\title{
Self-adaptive Search Equation-Based Artificial Bee Colony Algorithm with CMA-ES on the Noiseless BBOB Testbed*
}

\author{
Draft version
}

\author{
Dogan Aydin \\ Dumlupinar University \\ Computer Engineering Dept. 43000 \\ Kutahya, Turkey \\ dogan.aydin@dpu.edu.tr
}

\author{
Gurcan Yavuz \\ Dumlupinar University \\ Computer Engineering Dept. 43000 \\ Kutahya, Turkey \\ gurcan.yavuz@dpu.edu.tr
}

\begin{abstract}
Self-Adaptive Search Equation based Artificial Bee Colony (SSEABC) is a recent variant of Artificial Bee Colony $(A B C)$ algorithm. SSEABC proposed three enhancements on the canonical $A B C$ algorithm. These are the self-adaptive search equation selection strategy, hybridization with a local search procedure and incremental population size strategy. The performance of SSEABC is tested on CEC 2015 benchmark suite and ranked third within all participants of competition. In this paper, we benchmarks SSEABC using the noisefree $\mathrm{BBOB}$ function testbed. We also compare SSEABC performance to $\mathrm{PSO}, \mathrm{ABC}$ and GA algorithms.
\end{abstract}

\section{CCS CONCEPTS}

•Computing methodologies $\rightarrow$ Continuous space search;

\section{KEYWORDS}

Artificial Bee Colony, Continuous Domains, Self-Adaptation, Benchmarking

\section{ACM Reference format:}

Dogan Aydin and Gurcan Yavuz. 2017. Self-adaptive Search EquationBased Artificial Bee Colony Algorithm with CMA-ES on the Noiseless BBOB Testbed. In Proceedings of the Genetic and Evolutionary Computation Conference 2017, Berlin, Germany, fuly 15-19, 2017 (GECCO '17), 8 pages. DOI: $10.475 / 123 \_4$

\section{INTRODUCTION}

Ever since the Artificial Bee Colony $(\mathrm{ABC})$ algorithm came into existence [11], it has been used in solving continuous optimization problems. However, failure to produce successful results in some types of problems has led to the emergence of many improved $A B C$ variants in recent years. Many of these algorithms have suggested enhancements over one or more of the steps of the $\mathrm{ABC}$ algorithm $[1,3]$. A recent research [1] has shown that the best improvements can be made with changes to the empoyed bees and onlooker bees steps or with new extensions to the canonical ABC algorithm.

*Submission deadline: March 31st.

Permission to make digital or hard copies of part or all of this work for personal or classroom use is granted without fee provided that copies are not made or distributed for profit or commercial advantage and that copies bear this notice and the full citation on the first page. Copyrights for third-party components of this work must be honored. For all other uses, contact the owner/author(s).

GECCO '17, Berlin, Germany

(c) 2017 Copyright held by the owner/author(s). $123-4567-24-567 / 08 / 06 \ldots \$ 15.00$ DOI: $10.475 / 123 \_4$
A recent $\mathrm{ABC}$ variant, Self-adaptive Search Equation based Artificial Bee Colony (SSEABC) [14], is focused on these remediation methods. The SSEABC algorithm solves the problem of finding the appropriate search equation in the employed bees and onlooker bees steps in a self-adaptive way. On the other hand, the algorithm has been improved with iteratively increasing the number of populations and using local search procedures.

SSEABC algorithm performance has been compared with $A B C$ and many contemporary algorithms on CEC 2016 benchmark functions suite and it has been observed that we has obtained successful results [14]. In this paper, the performance of SSEABC algorithm on the BBOB functions testbed has been tested.

\section{ALGORITHM PRESENTATION}

SSEABC proposes three modifications on the original $A B C$ algorithm to improve performance. These strategies are based on the self-adaptive search equation selection, hybridization with a local search procedure and increasing population size during execution.The pseudo-code of SSEABC are presented in Algorithm 1.

Self-adaptive search equation selection: In solving numerical optimization problems, the most important factor affecting the performance of $\mathrm{ABC}$ algorithm is the search equations that take place in the steps of employed bees and onlooker bees. In addition to the search equation, the number of dimensions considered to be changed is another important factor affecting the performance of the algorithm. When considering the structure of the problem and that is supposed to be solved; determining the appropriate search equation becomes a difficult task. Thus, in this study, a mechanism has been developed that determines the appropriate search equation among the various candidates. To do this, SSEABC has proposed a search equation pool which is filled with randomly generated search equations. The general template of candidate search equation is as seen in Algorithm 2.

The candidate search equations take the form of four terms with alternatives in Table 1 with $m$ values. At initialization step of the algorithm, the pool, $S$, is filled by randomly generated search equation using Algorithm 2 and Table 1.Then, at each iteration a candidate search equation from the pool is used in the employed bees and onlooker bees steps. This process repeats until all candidates used in the pool. Throughout these steps, the number of success rates of the search equations that provide the update of the solution is increased. After all the candidate search equations in the pool are used in the employed bees and onlooker steps, $p s$, which is the size 


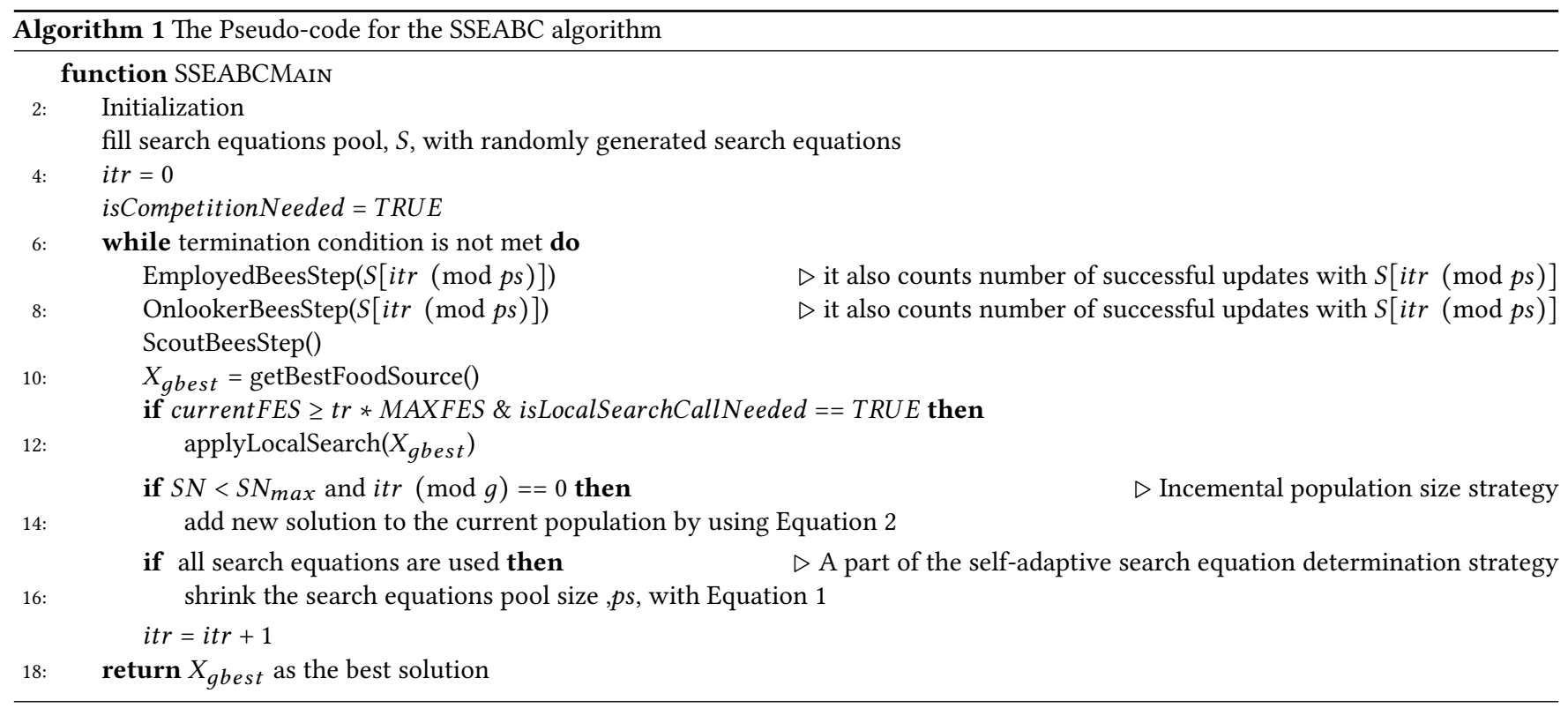

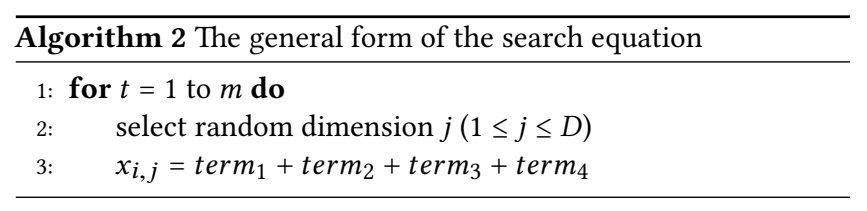

of the pool, is scaled down by the equation 1 :

$$
p s=\frac{p s^{2}}{i \operatorname{tr}_{M A X}}
$$

where MAXFES is the maximum number of function evaluations for one execution and $2 \times S N$ is the number of function evaluations at each iteration and where $\operatorname{itr}_{M A X}$ is the approximated value of the maximum number of iterations. tr $_{M A X}$ is the approximated value because the incremental population size strategy in which $S N$ is changing over time. Finally, when the algorithm finishes its execution, very few search equations, which are the appropriate ones, remain in the pool only.

hybridization with a local search procedure. : In SSEABC algorithm, bees move using the SSEABC rules and by the invocation of a local search procedure. Specifically, best-so-far solution is used as the initial solution a local search procedure is called from. The final solution found through local search becomes the new best-so-far solution if it is better than the initial solution. In SSEABC, the local search procedure is not called every iteration. The local search procedure is called only when it is expected that its invocation will result in an improvement of the-best-so-far solution. In previous implementation of SSEABC [14], competitive local search selection procedure was used. However, for the BBOB testbed, we used CMA-ES algorithm [12] as the local search procedure because competitive local search selection provides a wasteful use of function evaluations.

Incremental population size strategy: This strategy is very similar to the incremental social learning (ISL) framework [2, 4]. According
Table 1: Alternative options for each component in the generalized search equation. $x_{G, j}, x_{G D, j}, x_{S C, j}, x_{M D, j}, x_{W O, j}$ and $x_{A V E, j}$ are best-so-far, best-distance, second best, median, worst foods sources at dimension $j$, respectively. On the other hand, $X_{r 1}$ and $X_{r 2}$ are two randomly selected food source and $x_{A V E}, j$ refers to average positions of the food source at dimension $j . \phi_{N}$ can take two possible ranges: $[-1,-1]$ and $[-S F, S F]$ where $S F$ is randomly selected positive real value. These ranges are decided randomly while creating each component of randomly generated search equation.

\begin{tabular}{c|c|c}
\hline$m$ & term1 & term2 $\|$ terms $3 \|$ terms4 \\
\hline 1 & $x_{i, j}$ & $\phi N\left(x_{i, j}-x_{G, j}\right)$ \\
{$[t, k](1 \leq t<k \leq D)$} & $x_{G, j}$ & $\phi N\left(x_{i, j}-x_{r 1, j}\right)$ \\
& $x_{r 1, j}$ & $\phi N\left(x_{G, j}-x_{r 1, j}\right)$ \\
& & $\phi N\left(x_{r 1, j}-x_{r 2, j}\right)$ \\
& $\phi N\left(x_{i, j}-x_{G D, j}\right)$ \\
& $\phi N\left(x_{i, j}-x_{S C, j}\right)$ \\
& $\phi N\left(x_{i, j}-x_{M D, j}\right)$ \\
& $\phi N\left(x_{i, j}-x_{W O, j}\right)$ \\
& & $\phi N\left(x_{S C, j}-x_{M D, j}\right)$ \\
& & $\phi N\left(x_{M D, j}-x_{W O, j}\right)$ \\
& $\phi N\left(x_{G, j}-x_{W O, j}\right)$ \\
& $\phi N\left(x_{r 1, j}-x_{M D, j}\right)$ \\
& $\phi N\left(x_{G, j}-x_{M D, j}\right)$ \\
& $\phi N\left(x_{r 1, j}-x_{W O, j}\right)$ \\
& $\phi N\left(x_{S C, j}-x_{r 1, j}\right)$ \\
& & $\phi N\left(x_{i, j}-x_{A V E, j}\right)$ \\
& & $\phi N\left(x_{r 1, j}-x_{A V E, j}\right)$ \\
& & $\phi N\left(x_{G, j}-x_{A V E, j}\right)$ \\
& & DoNotUse \\
\hline
\end{tabular}


to this strategy, SSEABC starts to work with a small population. During the algorithm execution, a new solution influenced by the best-so-far solution is added to the population after a certain number of iterations called growth period, $g$. This addition process continues until the maximum population value is reached. The solution to be newly added to the population is created using the equation 2 :

$$
\dot{x}_{n e w, j}=x_{n e w, j}+\varphi_{i, j}\left(x_{G, j}-x_{n e w, j}\right)
$$

where $\dot{x}_{n e w, j}$ is the new solution to be added.

\section{EXPERIMENTAL PROCEDURE}

We have used the default parameter values for SSEABC and CMAES algorithms which were given in [14] and [12] respectively. A maximum of $10^{4} D$ function evaluations was used. Every periodic $2500 D$ function evaluations SSEABC restarts without forgetting the bestso-far solution.

\section{CPU TIMING}

In order to evaluate the CPU timing of the algorithm, we have run the SSEABC on the $f 8$ without restarts 30 seconds and until a maximum budget equal to $1000 \mathrm{D}$ is reached. The $\mathrm{C}++$ code was run on a Intel Xeon E5410 quadcore CPUs running at $2.33 \mathrm{GHz}$ with 2 $\mathrm{x} 6 \mathrm{MB}$ L2 cache and 8 GB RAM. The time per function evaluation for dimensions $2,3,5,10,20$, 40 equals $0.0041,0.0082,0.0146,0.627$, 1.421 , and 2,751 seconds respectively.

\section{RESULTS}

Results from experiments according to [10] and [6] on the benchmark functions given in [5,9] are presented in Figures 1, 2 and 3 and in Tables 2 and 3. The experiments were performed with $\mathrm{COCO}$ [8], version 2.0, the plots were produced with version 2.0.

The average runtime (aRT), used in the figures and tables, depends on a given target function value, $f_{\mathrm{t}}=f_{\mathrm{opt}}+\Delta f$, and is computed over all relevant trials as the number of function evaluations executed during each trial while the best function value did not reach $f_{\mathrm{t}}$, summed over all trials and divided by the number of trials that actually reached $f_{\mathrm{t}}[7,13]$. Statistical significance is tested with the rank-sum test for a given target $\Delta f_{\mathrm{t}}$ using, for each trial, either the number of needed function evaluations to reach $\Delta f_{\mathrm{t}}$ (inverted and multiplied by -1 ), or, if the target was not reached, the best $\Delta f$-value achieved, measured only up to the smallest number of overall function evaluations for any unsuccessful trial under consideration.

From the experiments, we observed that SSEABC solved 11 functions in dimension 5 and 5 functions in dimension 20 with $100 \%$ success rate. Over from dimension from 2 to 20 , SSEABC solved $f 1$, $f 5, f 6, f 7, f 8, f 9, f 11, f 12$ and $f 21$. For only $f 4, f 19$ and $f 24$, the algorithm can not reach any optimum value for dimension 5 and 20 .

Comparison of SSEABC algorithm to PSO, ABC and GA in previous $\mathrm{BBOB}$ workshops are presented in Figure 2. We have seen that SSEABC outperforms PSO, $A B C$ and GA for almost all functions. Moreover, SSEABC obtains better run-time performance than reference algorithms on the moderate, ill-conditioned and multi-modal functions.

\section{CONCLUSION}

In this paper, we present the benchmark results of SSEABC algorithm on $\mathrm{BBOB}$ functions testbed. We have also compared the performance of SSEABC to the data obtained by PSO, ABC and GA algorithms. The comparison results showed that SSEABC algorithm can outperforms the compared algorithms and it is very competitive to (1+1)-CMA-ES and BIPOP-CMA-ES in moderate and ill-conditioned functions.

\section{REFERENCES}

[1] Doğan Aydın. 2015. Composite artificial bee colony algorithms: From componentbased analysis to high-performing algorithms. Applied Soft Computing 32 (2015), 266-285.

[2] Doğan Aydın, Tianjun Liao, Marco A Montes de Oca, and Thomas Stützle. 2011. Improving performance via population growth and local search: the case of the artificial bee colony algorithm. In International Conference on Artificial Evolution (Evolution Artificielle). Springer, 85-96.

[3] Doğan Aydın, Gürcan Yavuz, and Thomas Stützle. 2017. ABC-X: a generalized, automatically configurable artificial bee colony framework. Swarm Intelligence 11,1 (2017), 1-38.

[4] Marco A Montes de Oca, Doğan Aydın, and Thomas Stützle. 2011. An incremental particle swarm for large-scale continuous optimization problems: an example of tuning-in-the-loop (re) design of optimization algorithms. Soft Computing 15, 11 (2011), 2233-2255.

[5] S. Finck, N. Hansen, R. Ros, and A. Auger. 2009. Real-Parameter Black-Box Optimization Benchmarking 2009: Presentation of the Noiseless Functions. Technical Report 2009/20. Research Center PPE. http://coco.lri.fr/downloads/download15. 03/bbobdocfunctions.pdf Updated February 2010.

[6] N. Hansen, A Auger, D. Brockhoff, D. Tušar, and T. Tušar. 2016. COCO: Performance Assessment. ArXiv e-prints arXiv:1605.03560 (2016).

[7] N. Hansen, A. Auger, S. Finck, and R. Ros. 2012. Real-Parameter Black-Box Optimization Benchmarking 2012: Experimental Setup. Technical Report. INRIA. http://coco.gforge.inria.fr/bbob2012-downloads

[8] N. Hansen, A. Auger, O. Mersmann, T. Tušar, and D. Brockhoff. 2016. COCO: A Platform for Comparing Continuous Optimizers in a Black-Box Setting. ArXiv e-prints arXiv:1603.08785 (2016).

[9] N. Hansen, S. Finck, R. Ros, and A. Auger. 2009. Real-Parameter Black-Box Optimization Benchmarking 2009: Noiseless Functions Definitions. Technical Report RR6829. INRIA. http://coco.lri.fr/downloads/download15.03/bbobdocfunctions.pdf Updated February 2010.

[10] N. Hansen, T. Tušar, O. Mersmann, A. Auger, and D. Brockhoff. 2016. COCO: The Experimental Procedure. ArXiv e-prints arXiv:1603.08776 (2016).

[11] Dervis Karaboga and Bahriye Basturk. 2007. A powerful and efficient algorithm for numerical function optimization: artificial bee colony $(\mathrm{ABC})$ algorithm. Journal of global optimization 39, 3 (2007), 459-471.

[12] Václav Klemøs Petr Poøsík. 2012. Benchmarking the Differential Evolution with Adaptive Encoding on Noiseless Functions. In GECCO 2012 (Companion), Terence Soule (Ed.). ACM.

[13] Kenneth Price. 1997. Differential evolution vs. the functions of the second ICEO. In Proceedings of the IEEE International Congress on Evolutionary Computation. 153-157.

[14] Gurcan Yavuz, Doğan Aydin, and Thomas Stützle. 2016. Self-adaptive search equation-based artificial bee colony algorithm on the CEC 2014 benchmark functions. In Evolutionary Computation (CEC), 2016 IEEE Congress on. IEEE, 11731180 . 

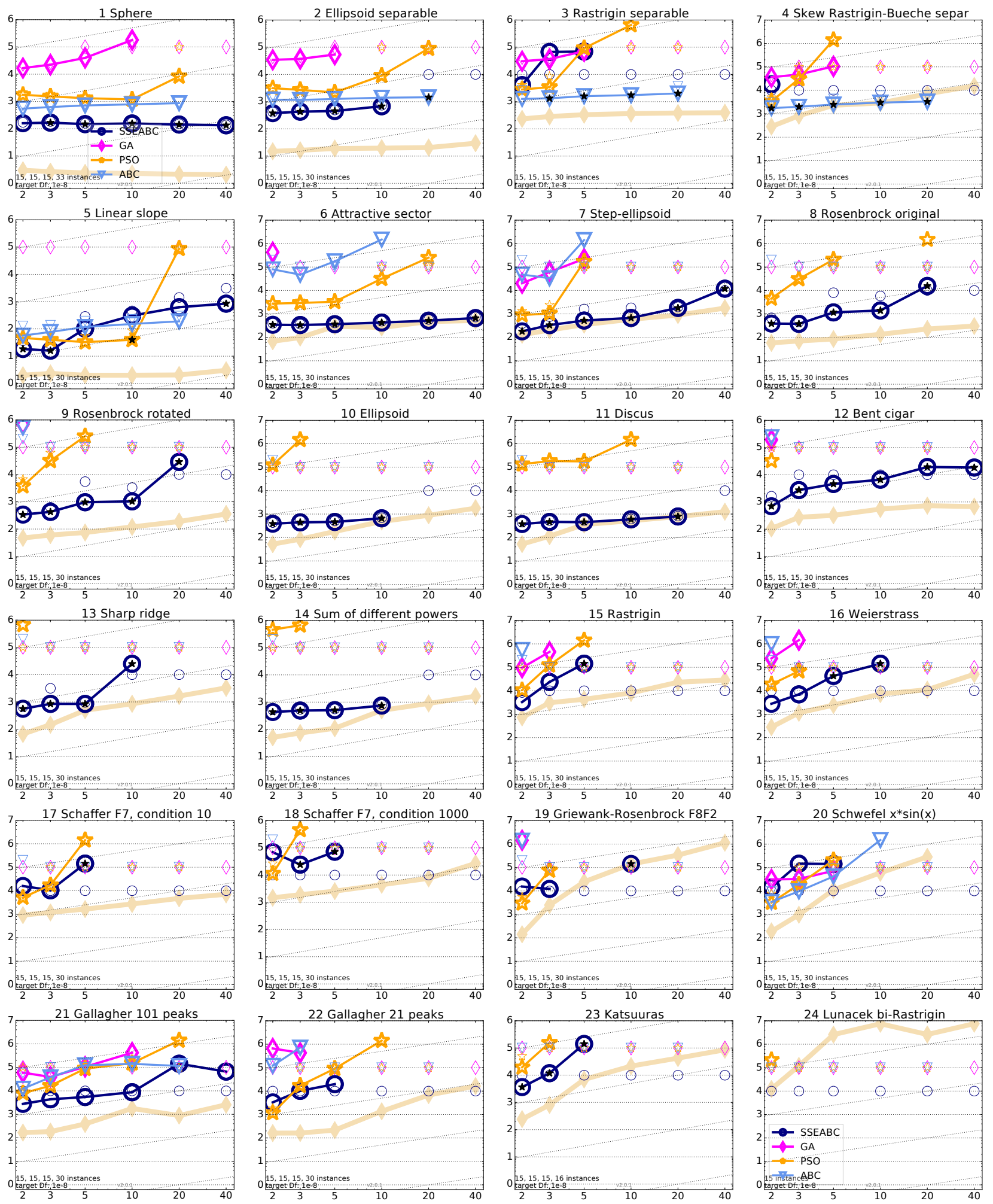

Figure 1: Average running time (aRT in number of $f$-evaluations as $\log _{10}$ value), divided by dimension for target function value $10^{-8}$ versus dimension. Slanted grid lines indicate quadratic scaling with the dimension. Different symbols correspond to different algorithms given in the legend of $f_{1}$ and $f_{24}$. Light symbols give the maximum number of function evaluations from the longest trial divided by dimension. Black stars indicate a statistically better result compared to all other algorithms with $p<0.01$ and Bonferroni correction number of dimensions (six). Legend: $\circ$ SSEABC, $\diamond:$ GA, $\star$ : PSO, $\nabla:$ ABC 
separable fcts

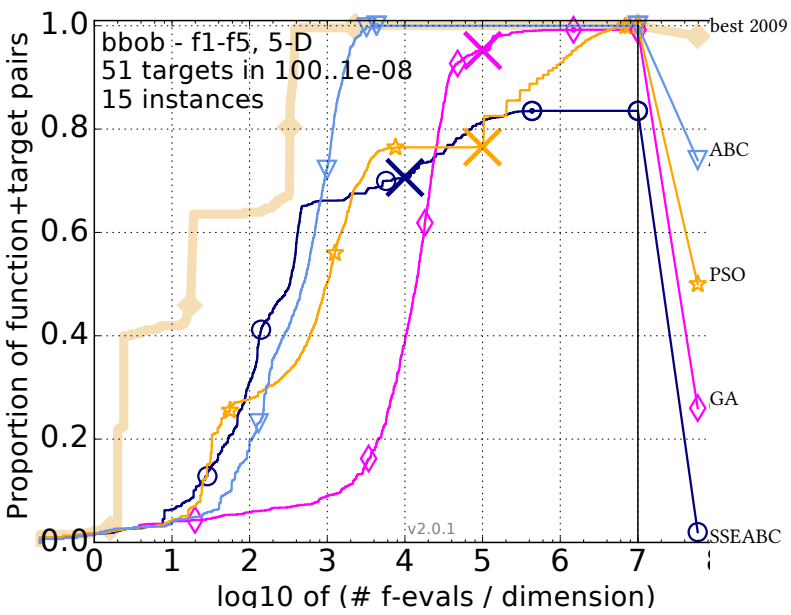

ill-conditioned fcts
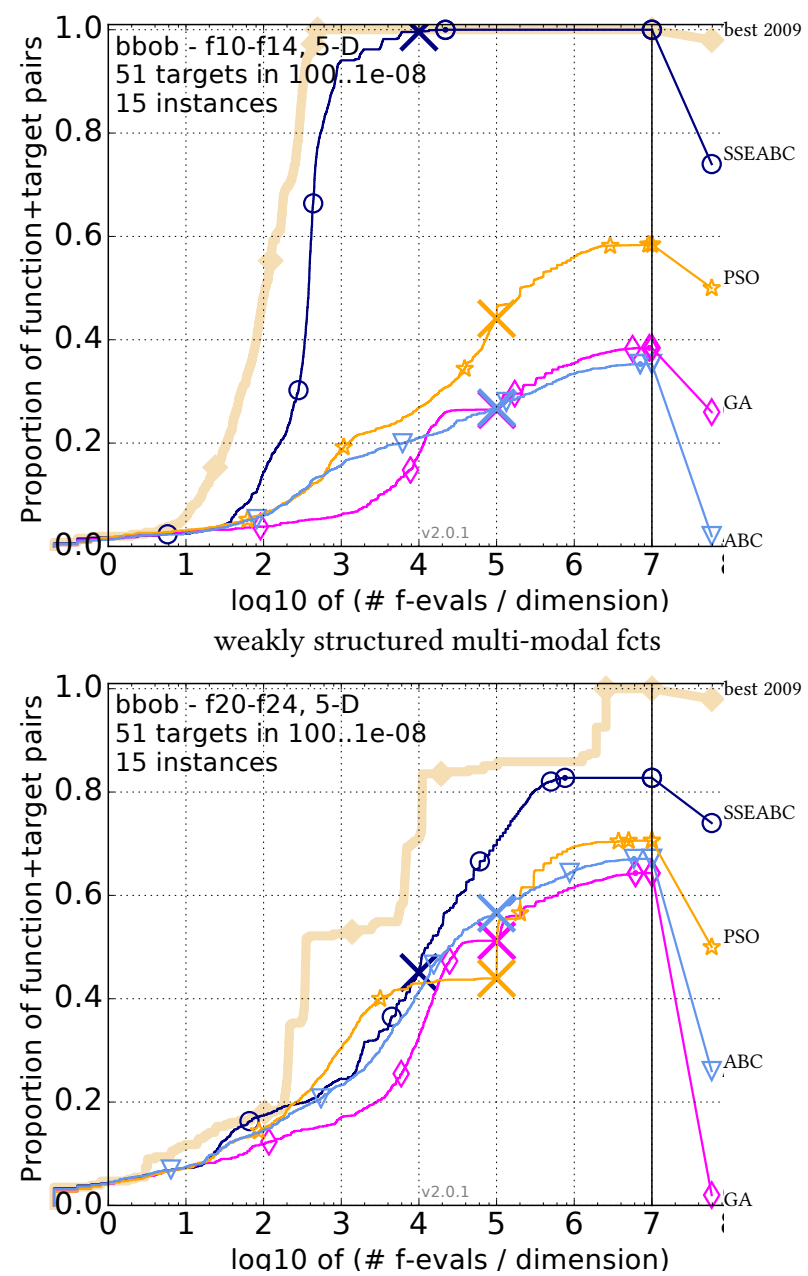

moderate fcts

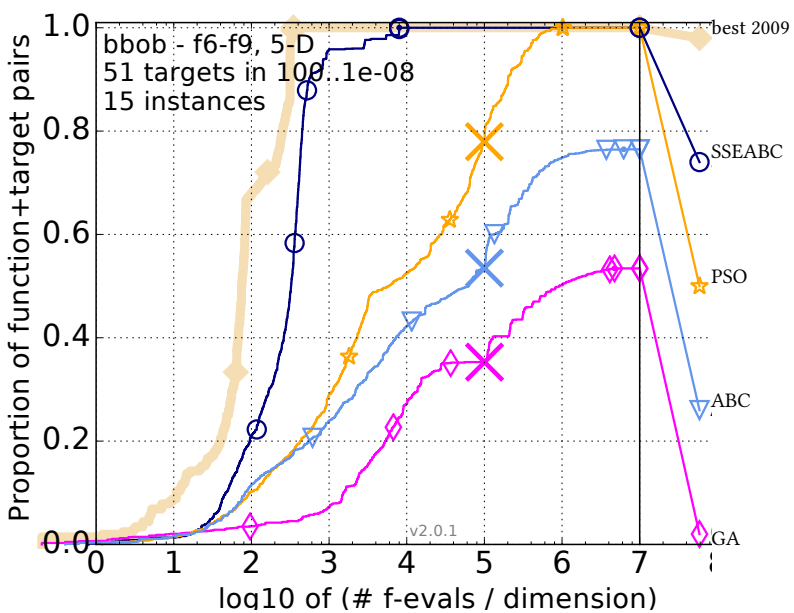
multi-modal fcts

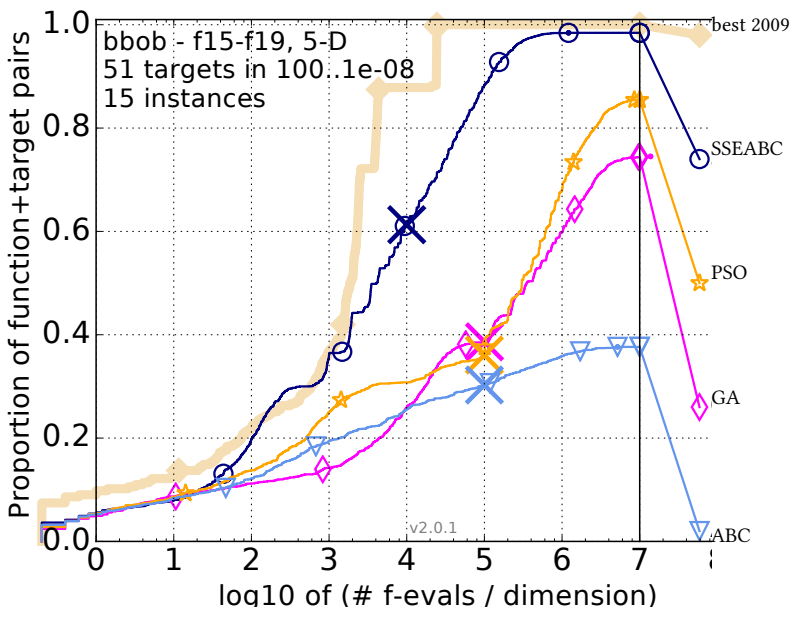
all functions

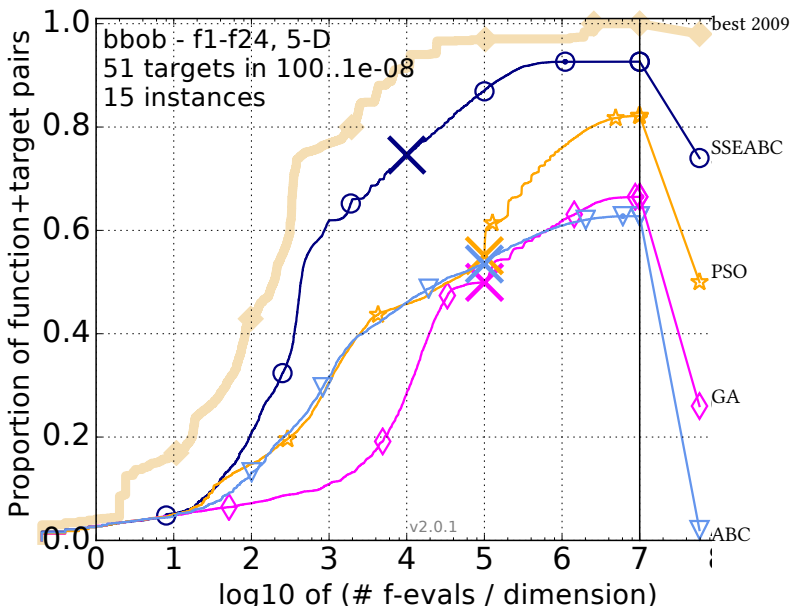

Figure 2: Bootstrapped empirical cumulative distribution of the number of objective function evaluations divided by dimension (FEvals/DIM) for 51 targets with target precision in $10^{[-8 . .2]}$ for all functions and subgroups in 5-D. The "best 2009" line corresponds to the best aRT observed during BBOB 2009 for each selected target. 
separable fcts

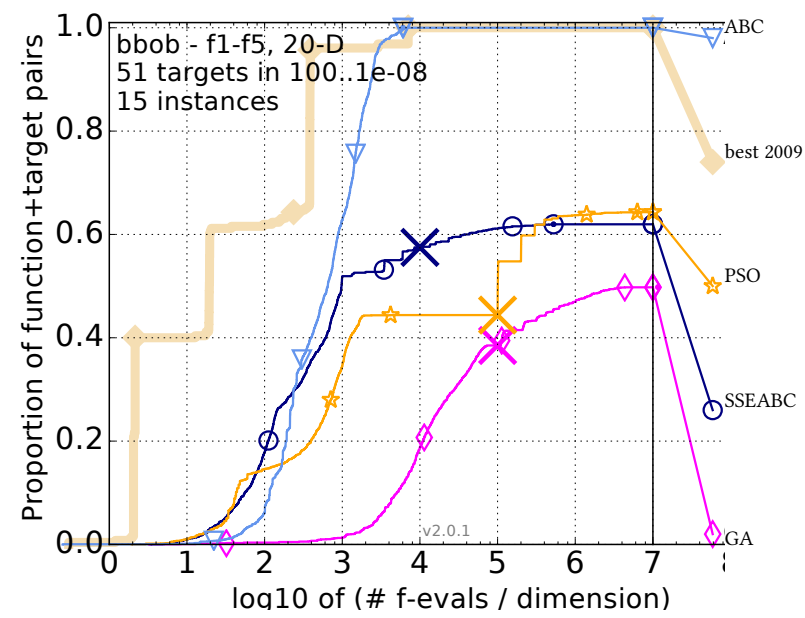

ill-conditioned fcts
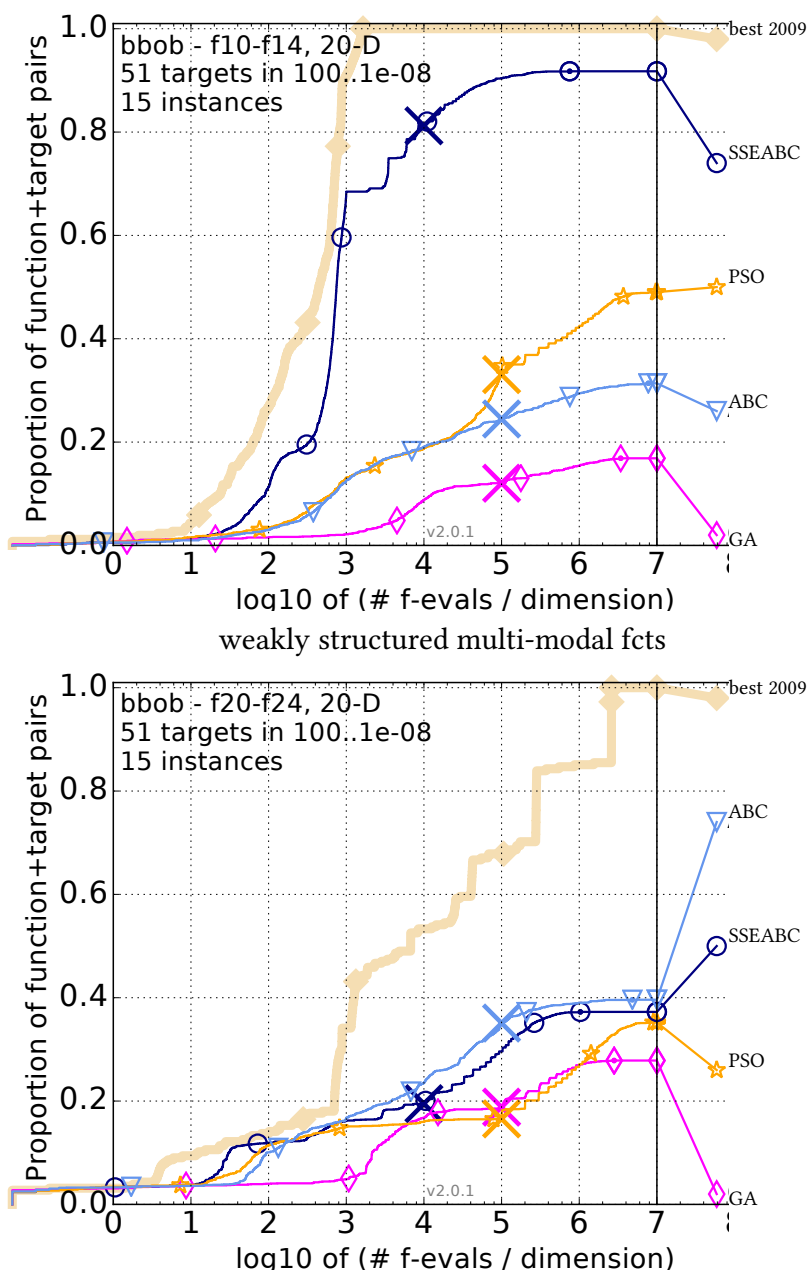

moderate fcts

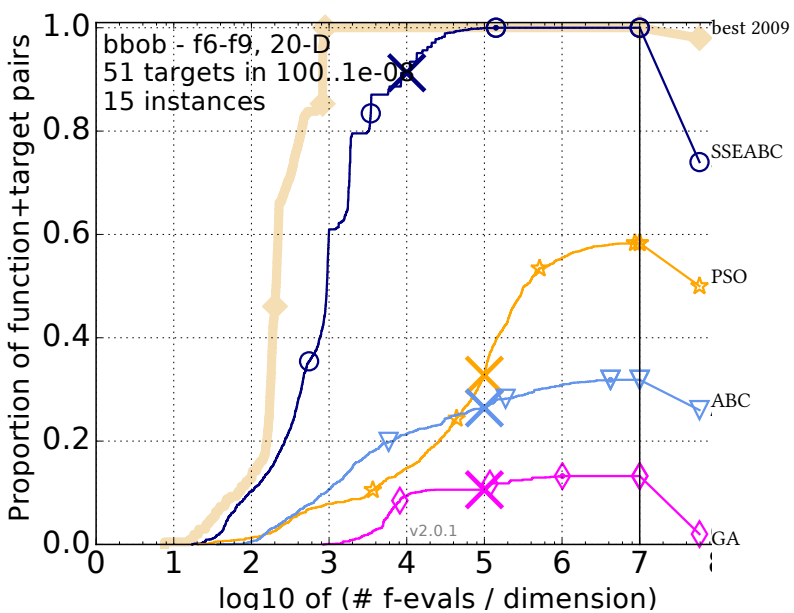
multi-modal fcts

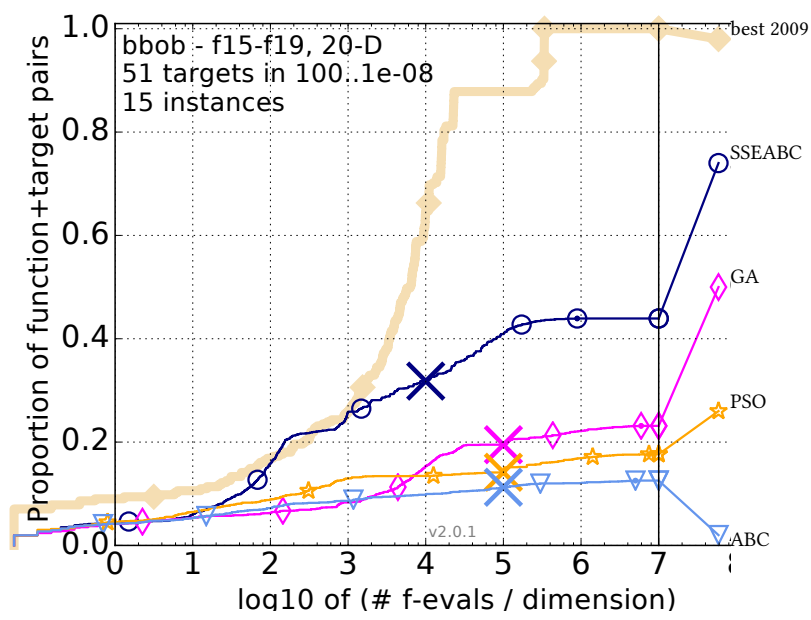

all functions

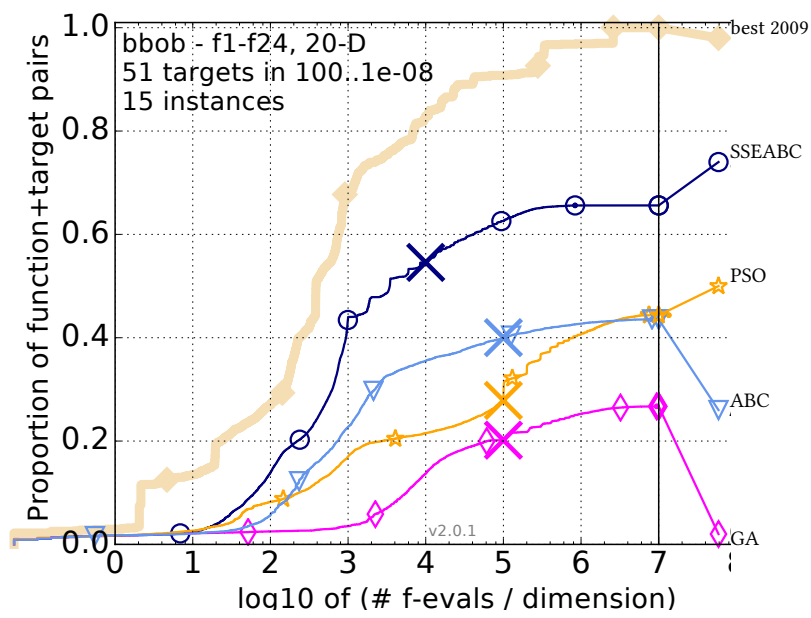

Figure 3: Bootstrapped empirical cumulative distribution of the number of objective function evaluations divided by dimension (FEvals/DIM) for 51 targets with target precision in $10^{[-8 . .2]}$ for all functions and subgroups in 20-D. The "best 2009" line corresponds to the best $\mathrm{aRT}$ observed during $\mathrm{BBOB} 2009$ for each selected target. 


\begin{tabular}{|c|c|c|c|c|c|c|c|c|c|c|c|c|c|c|c|c|c|}
\hline$\Delta f_{\text {opt }}$ & |e1 & 1e0 & $1 \mathrm{e}-1$ & $1 e-2$ & $1 e-3$ & $1 e-5$ & $1 e-7$ & \#succ & $\Delta f_{\text {opt }}$ & $1 \mathrm{e} 1$ & $1 \mathrm{e} 0$ & $1 \mathrm{e}-1$ & $1 \mathrm{e}-2$ & $1 e-3$ & $1 e-5$ & $1 e-7$ & $\#$ succ \\
\hline f1 & 11 & 12 & 12 & 12 & 12 & 12 & 12 & $15 / 15$ & f13 & 132 & 195 & 250 & 319 & 1310 & 1752 & 2255 & $15 / 15$ \\
\hline SSEABC & $5.9(1)$ & $12(1)^{\star 2}$ & $18(3)^{\star 3}$ & $26(5)^{\star 4}$ & $32(2)^{\star 4}$ & $45(4)^{\star 4}$ & $56(2)^{\star 4}$ & $15 / 15$ & SSEABC & $3.8(3)^{\star 2}$ & $5.9(3)^{\star 4}$ & $6.5(3)^{\star}$ & $6.7(3)^{\star 4}$ & $1.9(0.4)^{\star 4}$ & $1.8(0.3)^{\star 4}$ & $1.8(0.2)^{\star 4}$ & $15 / 15$ \\
\hline A & $8.7(6)$ & $369(261)$ & 1202(181) & 2130(381) & $2989(437)$ & $5474(453)$ & $8468(960)$ & $13 / 15$ & $\mathrm{GA}$ & $243(26)$ & $726(672)$ & $4390(3487)$ & $2.3 \mathrm{e} 4(4 \mathrm{e} 4$ & $\infty$ & $\infty$ & $\infty 5 e 5$ & $0 / 15$ \\
\hline PSO & $3.7(2)$ & $22(6)$ & (20) & $7(22)$ & $5(31)$ & 2(56) & $8(42)$ & $15 / 15$ & PSO & $1582(1894)$ & $.0 \mathrm{e} 4(2 \mathrm{e} 4)$ & $2.8 \mathrm{e} 4(3 \mathrm{e} 4)$ & $\infty$ & $\infty$ & $\infty$ & $\infty 5 e 5$ & $0 / 15$ \\
\hline $\mathrm{ABC}$ & $12(14)$ & $32(20)$ & 63(18) & $90(22)$ & $124(29)$ & $194(23)$ & $259(9)$ & $15 / 15$ & $\mathrm{ABC}$ & $18(16)$ & $187(109)$ & 6613(9121) & $\infty$ & $\infty$ & $\infty$ & $\infty 5 e 5$ & $0 / 15$ \\
\hline$\Delta f_{\mathrm{opt}}$ & 1e1 & $1 \mathrm{e} 0$ & $1 \mathrm{e}-1$ & $1 \mathrm{e}-2$ & $1 e-3$ & $1 e-5$ & $1 e-7$ & \#succ & $\Delta f_{\text {opt }}$ & $1 \mathrm{e} 1$ & $1 \mathrm{e} 0$ & $1 e-1$ & $1 e-2$ & $1 e-3$ & $1 e-5$ & $1 \mathrm{e}-7$ & \#succ \\
\hline f2 & 83 & 87 & 88 & 89 & 90 & 92 & 94 & $15 / 15$ & f14 & 10 & 41 & 58 & 90 & 139 & 251 & 476 & $15 / 15$ \\
\hline SSEABC & $15(5)$ & $18(3)$ & $19(2)$ & $20(1)$ & $21(2)^{\star 3}$ & $22(2)^{\star 4}$ & $23(2)^{\star 4}$ & $15 / 15$ & SSEABC & c $3.0(3)$ & $3.7(0.7)$ & $4.4(0.9)^{\star 4}$ & $4.4(0.6)^{\star 4}$ & $4.8(0.7)^{\star 4}$ & $5.7(0.5)^{\star 4}$ & $4.6(0.3)^{\star 4}$ & $15 / 15$ \\
\hline $\mathrm{GA}$ & $334(44)$ & $459(50)$ & $609(68)$ & 772(73) & $1301(2806)$ & $2156(108)$ & $2535(2796)$ & $13 / 15$ & $\mathrm{GA}$ & 2.1(1) & $90(56)$ & $267(68)$ & $305(32)$ & $349(59)$ & $\infty$ & $\infty 5 e 5$ & $0 / 15$ \\
\hline so & $32(8)$ & $41(7)$ & $49(7)$ & 11) & $68(11)$ & $89(11)$ & $106(9)$ & $15 / 15$ & PSO & $1.8(2)$ & $5.6(3)$ & $15(3)$ & $21(7)$ & $29(8)$ & $219(188)$ & $\infty 5 e 5$ & $0 / 15$ \\
\hline $\mathrm{ABC}$ & $11(5)$ & $18(6)$ & $26(10)$ & $30(13)$ & $38(11)$ & $50(11)$ & $62(7)$ & $15 / 15$ & $\mathrm{ABC}$ & $3.4(3)$ & $11(7)$ & $19(6)$ & $29(8)$ & $677(681)$ & & $\infty 5 e 5$ & $0 / 15$ \\
\hline$\Delta f_{\text {opt }}$ & 1e1 & $1 \mathrm{e} 0$ & $1 e-1$ & $1 \mathrm{e}-2$ & $1 e-3$ & $1 e-5$ & $1 e-7$ & \#succ & $\Delta f_{\mathrm{opt}}$ & $1 \mathrm{e} 1$ & $1 \mathrm{e} 0$ & $1 e-1$ & $1 \mathrm{e}-2$ & $1 e-3$ & $1 e-5$ & $1 e-7$ & \#succ \\
\hline f3 & 716 & 1622 & 1637 & 1642 & 1646 & 1650 & 1654 & $15 / 15$ & f15 & 511 & 9310 & 19369 & 19743 & 20073 & 20769 & 21359 & $14 / 15$ \\
\hline SSEABC & $\begin{array}{l}=1.2(1.0) \\
\end{array}$ & $9.2(7)$ & $49(66)$ & $74(63)$ & $132(206)$ & 2(109) & $.0(257)$ & $2 / 15$ & SSEABC & c $1.2(0.2)^{\star 3}$ & $0.93(0.5)^{\star 3}$ & $1.0(0.9)^{\star 4}$ & $1.1(1.0)^{\star 4}$ & $1.7(0.9)^{\star 4}$ & $7.9(7)^{\star 4}$ & $10(6)^{\star 4}$ & $1 / 15$ \\
\hline $\mathrm{GA}$ & $19(2)$ & $18(0.8)$ & $25(2)$ & $34(4)$ & $43(4)$ & $112(155)$ & $200(155)$ & $11 / 15$ & $\begin{array}{l}\text { SSEABC } \\
\text { GA }\end{array}$ & $35(6)$ & $91(122)$ & $367(454)$ & $361(540)$ & $355(431)$ & $345(254)$ & $\infty 5 e 5$ & $0 / 15$ \\
\hline PSO & $52(2)$ & $55(156)$ & $275(614)$ & $275(457)$ & $275(610)$ & $276(456)$ & $276(532)$ & $8 / 15$ & PSO & $16(72)$ & $221(284)$ & 366 & $359(266)$ & $353(380)$ & $342(391)$ & $333(380)$ & $1 / 15$ \\
\hline $\mathrm{ABC}$ & 1.0(0.4) & $1.5(0.8)^{\star 3}$ & $\mathbf{1 . 8}(0.6)^{\star 4}$ & $2.4(0.4)^{\star 4}$ & $2.7(0.4)^{\star 4}$ & $\mathbf{3 . 6}(0.4)^{\star 4}$ & $\mathbf{4 . 4}(0.7)^{\star 4}$ & $15 / 15$ & $\mathrm{ABC}$ & $15(4)$ & $243(497)$ & $\infty$ & $\infty$ & & & $\infty 5 e 5$ & $0 / 15$ \\
\hline$\Delta f_{\text {opt }}$ & | $1 \mathrm{e} 1$ & $1 \mathrm{e} 0$ & $1 e-1$ & $1 e-2$ & $1 \mathrm{e}-3$ & $1 e-5$ & $1 e-7$ & $\#$ succ & $\Delta f_{\mathrm{opt}}$ & 1e1 & $1 \mathrm{e} 0$ & $1 \mathrm{e}-1$ & $1 \mathrm{e}-2$ & $1 e-3$ & $1 e-5$ & $1 e-7$ & \#succ \\
\hline f4 & 809 & 1633 & 1688 & 1758 & 1817 & 1886 & 1903 & $15 / 15$ & f16 & 120 & 612 & 2662 & 10163 & 10449 & 11644 & 12095 & $15 / 15$ \\
\hline SEABC & $2.7(3)$ & $\infty$ & $\infty$ & $\infty$ & $\infty$ & $\infty$ & $65 e 4$ & $0 / 15$ & SSEABC & d2.8(2) & $2.0(4)^{\star}$ & 4.1(2) & $1.4(0.6)^{\star}$ & $1.9(2) \star 2$ & $3.8(5)^{\star 2}$ & $18(20) \star 2$ & $3 / 15$ \\
\hline GA & $18(3)$ & $20(3)$ & $26(2)$ & $33(5)$ & $41(5)$ & $58(10)$ & $185(269)$ & $9 / 15$ & $\mathrm{GA}$ & $2.1(2)$ & $84(53)$ & & $71(64)$ & $148(122)$ & $21(647)$ & $605(498)$ & $0 / 15$ \\
\hline PSO & $3.0(1.0)$ & $141(155)$ & 4152(3555) & 3988(2631) & $3859(5297)$ & $3719(3049)$ & $3687(2430)$ & $1 / 15$ & PSO & $2.4(4)$ & $6.2(8)$ & $59(112)$ & $55(89)$ & $\begin{array}{l}17(124) \\
89(69)\end{array}$ & $300(321)$ & $80(734)$ & $0 / 15$ \\
\hline $\mathrm{ABC}$ & 1.1(0.5) & $2.4(1)^{\star 3}$ & $2.9(0.7)^{\star 4}$ & $3.4(1)^{\star 4}$ & $4.3(1)^{\star 3}$ & $4.9(2)^{\star 4}$ & $6.0(1)^{\star 4}$ & $15 / 15$ & $A B C$ & $2.3(1)$ & $10(8)$ & $95(122)$ & $\infty$ & $\infty$ & $\infty$ & $\infty 5 e 5$ & $0 / 15$ \\
\hline$\Delta f_{\text {opt }}$ & 1e1 & $1 \mathrm{e} 0$ & $1 e-1$ & $1 \mathrm{e}-2$ & $1 e-3$ & $1 e-5$ & $1 e-7$ & \#succ & $\Delta f_{\mathrm{opt}}$ & 1e1 & $1 \mathrm{e} 0$ & $1 e-1$ & $1 \mathrm{e}-2$ & $1 e-3$ & $1 e-5$ & $1 e-7$ & \#succ \\
\hline $\mathrm{f5}$ & 10 & 10 & 10 & 10 & 10 & 10 & 10 & $15 / 15$ & f17 & 5.0 & 215 & 899 & 2861 & 3669 & 6351 & 7934 & $15 / 15$ \\
\hline SSEABC & $6.6(3)^{\star 2}$ & $22(14$ & $34(29)$ & $41(56)$ & 46( & $51(50)$ & $51(41)$ & $15 / 15$ & SSEABC & c $4.4(5)$ & $1.1(0.1)^{\star 3}$ & $\mathbf{1 . 4}(0.1)^{\star 2}$ & $1.2(1)^{\star 2}$ & $1.2(1)^{\star 3}$ & $20(16)^{\star}$ & $90(49)^{\star}$ & $1 / 15$ \\
\hline $\mathrm{GA}$ & $481(168)$ & 2072 & 398 & 634 & $9220(696)$ & 1.7e $4(1601)$ & et $4(38$ & $0 / 15$ & $\mathrm{GA}$ & & 46 & $36(3)$ & $52(90)$ & 774) & $550(651)$ & & $0 / 15$ \\
\hline PSO & $10(3)$ & 14 & 1 & 16 & $16(9)$ & $16(7)$ & $16(8)$ & $15 / 15$ & PSO & 3.4(6) & 169 & $142(140)$ & $156(175)$ & $548(716)$ & $514(571)$ & 88) & $1 / 15$ \\
\hline $\mathrm{ABC}$ & $32(26)$ & $49(24)$ & $58(29)$ & $59(24)$ & $59(35)$ & $59(35)$ & $59(38)$ & $15 / 15$ & $\mathrm{ABC}$ & $6.8(9)$ & $15(23)$ & $64(68)$ & $1259(536)$ & $\infty$ & $\infty$ & $\infty 5 e 5$ & $0 / 15$ \\
\hline$\Delta f_{\text {opt }}$ & |1e1 & $1 \mathrm{e} 0$ & $1 e-1$ & $1 \mathrm{e}-2$ & $1 \mathrm{e}-3$ & $1 e-5$ & $1 e-7$ & \#succ & $\Delta f_{\text {opt }}$ & $1 \mathrm{e} 1$ & $1 \mathrm{e} 0$ & $1 e-1$ & $1 \mathrm{e}-2$ & $1 e-3$ & $1 e-5$ & $1 e-7$ & \#succ \\
\hline$f 6$ & 114 & 21 & 281 & 404 & 580 & 1038 & 13 & $15 / 15$ & f18 & 103 & 378 & 3968 & 8451 & 9280 & 10905 & 12469 & $15 / 15$ \\
\hline SSEABC & $2.0(0.3)^{\star 2}$ & $1.9(0.6)^{\star 4}$ & $2.0(0.3)^{\star 4}$ & $1.8(0.1)^{\star 4}$ & $\mathbf{1 . 6}(0.2)^{\star 4}$ & $1.2(0.2)^{\star 4}$ & $1.2(0.2)^{\star 4}$ & $15 / 15$ & SSEABC & c $1.5(0.5)$ & $2.2(2)^{\star 2}$ & $0.89(0.7)$ & $0.89(0.5)^{\star 3}$ & $3 \quad 1.4(1) \star 4$ & $21(26)^{\star 4}$ & $29(36)^{\star 4}$ & $2 / 15$ \\
\hline $\mathrm{GA}$ & $66(39)$ & $148(59)$ & $382(48)$ & $3680(5284)$ & $.2 \mathrm{e} 4(1 \mathrm{e} 4)$ & $\infty$ & $\infty 5 e 5$ & $0 / 15$ & $\mathrm{GA}$ & $22(14)$ & $59(8)$ & $34(34)$ & 34105 & $\infty$ & - & $\infty 5 e 5$ & $0 / 15$ \\
\hline PSO & $4.7(2)$ & $9.0(4)$ & 11(4) & $12(2)$ & $11(3)$ & $10(0.9)$ & (1) & $15 / 15$ & PSO & $2.3(2)$ & 6. & $113(189)$ & $253(417)$ & - & c & $\infty 5 e 5$ & $0 / 15$ \\
\hline $\mathrm{ABC}$ & $4.9(4)$ & $15(10)$ & $365(1360)$ & $408(953)$ & 619(1294) & $498(559)$ & $507(1126)$ & 6/15 & $\mathrm{ABC}$ & $5.1(4)$ & $27(25)$ & $300(174)$ & $\infty$ & $\infty$ & $\infty$ & $\infty 5 e 5$ & $0 / 15$ \\
\hline$\Delta f_{\text {opt }}$ & 1e1 & $1 \mathrm{e} 0$ & $1 \mathrm{e}-1$ & $1 e-2$ & $1 \mathrm{e}-3$ & $1 e-5$ & $1 \mathrm{e}-7$ & \#succ & $\Delta f_{\text {opt }}$ & 1e 1 & $1 \mathrm{e} 0$ & $1 e-1$ & $1 \mathrm{e}-2$ & $1 \mathrm{e}-3$ & $1 e-5$ & $1 e-7$ & \#succ \\
\hline f7 & 24 & 324 & 1171 & 1451 & 1572 & 1572 & 1597 & $15 / 15$ & f19 & 1 & 1 & 242 & $1.0 \mathrm{e} 5$ & $1.2 \mathrm{e} 5$ & $1.2 \mathrm{e} 5$ & $1.2 \mathrm{e} 5$ & $15 / 15$ \\
\hline SSEABC & $5.9(3)$ & $1.9(2)$ & $\mathbf{1 . 4}(0.7)^{\star 3}$ & $1.4(1.0)^{\star 3}$ & 1.4( $(0.8)^{\star 4}$ & $\mathbf{1 . 4}(1) \star 4$ & $\mathbf{1 . 4}(0.7)^{x}$ & $15 / 15$ & SSEABC & C $47(40)$ & $2379(1603)$ & $158(175)^{\star}$ & $3.4(5)^{\star 2}$ & $6.0(8)^{\star 2}$ & $6.0(5)^{\star}$ & $5.9(9) \star 2$ & $0 / 15$ \\
\hline & $49(53)$ & & $57(10)$ & $245(358)$ & $524(574)$ & & & $5 / 15$ & $\mathrm{GA}$ & $35(22)$ & & & & & $\infty$ & & $0 / 15$ \\
\hline SO & $11(14)$ & 9.5 & $587(855)$ & $475(3$ & 5 & 5 & & $6 / 15$ & PSO & 35 & & & & $60(5$ & $61(65$ & $61(8$ & o/15 \\
\hline $\mathrm{ABC}$ & $19(7)$ & $16(14)$ & $62(58)$ & 464(330) & $957(714)$ & $957(1173)$ & 1359(1418) & 1/15 & $\mathrm{ABC}$ & $34(34)$ & $2898(1832)$ & $3826(5335)$ & $69(130)$ & $\infty$ & $\infty$ & $\infty 5 e 5$ & $0 / 15$ \\
\hline$\Delta f_{\text {opt }}$ & 1e1 & $1 \mathrm{e} 0$ & $1 \mathrm{e}-1$ & $1 \mathrm{e}-2$ & $1 e-3$ & $1 e-5$ & $1 e-7$ & \#succ & $\Delta f_{\text {opt }}$ & 1e1 & $1 \mathrm{e} 0$ & $1 \mathrm{e}-1$ & $1 \mathrm{e}-2$ & $1 e-3$ & $1 e-5$ & $1 e-7$ & \#succ \\
\hline $\mathrm{f} 8$ & 73 & 273 & 336 & 372 & 391 & 410 & 42 & $15 / 15$ & $\mathrm{f} 20$ & 16 & 851 & 38111 & 51362 & 5447 & 54861 & 5531 & $14 / 15$ \\
\hline SSEABC & $4.7(1)$ & & $8.7(0.9)^{\star 2}$ & $14(34)^{\star 3}$ & $14(8)^{\star 4}$ & $14(46)^{\star 4}$ & $14(23)^{\star 4}$ & $15 / 15$ & SSEABC & C $6.9(4)$ & $12(7)$ & $18(21)$ & $14(21)$ & 13(15) & 13(38) & 13(14) & $1 / 15$ \\
\hline & $187(33)$ & & $\infty$ & $\infty$ & $\infty$ & $\infty$ & & $0 / 15$ & $\mathrm{GA}$ & $47(31)$ & $21(4)$ & & & & $2.6(0.2)$ & $5.0(0.3)$ & $11 / 15$ \\
\hline PSO & $13(3)$ & & $201(47)$ & $313(769)$ & 467 & $781(610)$ & $1104(107)$ & $7 / 15$ & PSO & $8.7(4)$ & $3.1(0.9)$ & $27(33)$ & $20(20)$ & $19(25)$ & 19(7) & $18(23)$ & $5 / 15$ \\
\hline $\mathrm{ABC}$ & $6.0(3)$ & $12(14)$ & $52(11)$ & $449(361)$ & $2510(1741)$ & $\infty$ & $\infty 5 e 5$ & | $0 / 15$ & $\mathrm{ABC}$ & $7.2(4)$ & $\mathbf{1 . 5}(0.8)^{\star 2}$ & $0.55(0.6)$ & $\mathbf{0 . 4 8}(0.4)$ & $0.58(0.4)$ & $\mathbf{1 . 5}(0.5)$ & $2.6(2)$ & $15 / 15$ \\
\hline$\Delta f_{\text {opt }}$ & | $1 \mathrm{e} 1$ & $1 \mathrm{e} 0$ & $1 e-1$ & $1 e-2$ & $1 \mathrm{e}-3$ & $1 e-5$ & $1 e-7$ & \#succ & $\Delta f_{\text {opt }}$ & 1e1 & $1 \mathrm{e} 0$ & $1 \mathrm{e}-1$ & $1 \mathrm{e}-2$ & $1 e-3$ & $1 e-5$ & $1 \mathrm{e}-7$ & \#succ \\
\hline f9 & 35 & 127 & 21 & 263 & 300 & 33 & & $15 / 15$ & f21 & 41 & 57 & & 169 & 170 & 1729 & 175 & $14 / 15$ \\
\hline SSEABC & $9.0(3)$ & $11(7)^{\star 3}$ & $9.0(2) \star 3$ & $\mathbf{1 4}(25)^{\star 3}$ & $15(11)^{\star 4}$ & $14(10)^{\star 4}$ & $13(0.7)^{\star 4}$ & $15 / 15$ & SSEABC & C2.1(1) & $5.0(9$ & 13( & 13( & 13( & $13(25)$ & $15(30)$ & $12 / 15$ \\
\hline & $418(149)$ & & $\propto$ & $\infty$ & $\infty$ & $\infty$ & $\infty$ & $0 / 15$ & GA & 4.6 & 5.5 & $61(\mathrm{~S}$ & & & & & $8 / 15$ \\
\hline PSC & $24(1$ & $938(1$ & $678(1760)$ & 794(1533) & $1131(1007)$ & 63 & & $5 / 15$ & PSO & 2.0 & $379(541)$ & $262(224)$ & $260(517)$ & $258(221)$ & $255(290)$ & & $8 / 15$ \\
\hline $\mathrm{ABC}$ & $14(8)$ & $69(22)$ & 699(961) & $3994(5152)$ & $\infty$ & $\infty$ & $\infty 5 e 5$ & $0 / 15$ & $\mathrm{ABC}$ & $3.2(0.8)$ & $1.8(2)$ & $6.7(7)$ & $10(9)$ & 13(9) & $84(108)$ & $265(305)$ & $8 / 15$ \\
\hline$\Delta f_{\text {opt }}$ & 1e1 & $1 \mathrm{e} 0$ & 1e-1 & $1 \mathrm{e}-2$ & $1 e-3$ & $1 e-5$ & $1 e-7$ & \#succ & $\Delta f_{\mathrm{opt}}$ & 1e1 & $1 \mathrm{e} 0$ & $1 \mathrm{e}-1$ & $1 \mathrm{e}-2$ & $1 e-3$ & $1 e-5$ & $1 e-7$ & \#succ \\
\hline f10 & 349 & 500 & 574 & 607 & 62 & 829 & 88 & $15 / 15$ & f22 & 71 & 386 & 938 & 980 & 1008 & 1040 & 1068 & $14 / 15$ \\
\hline SSEABC & $3.5(1)^{\star 4}$ & & $3.0(0$ & $\mathbf{3 . 0}(0.3)^{\star 4}$ & & & & & SSEABC & c $3.2(5)$ & $40(126)$ & $51(50)$ & $60(44)$ & $68(50)$ & $81(56)$ & 93(137) & $6 / 15$ \\
\hline & $\begin{array}{c}3 .(1) \\
2375(3238)\end{array}$ & $\infty^{3}$ & 3.00 & 3.00 & $\infty_{\infty}^{.10}$ & $\infty$ & $\begin{array}{l}2.5(0.3) \\
\infty 5 e 5\end{array}$ & $\begin{array}{l}15 / 15 \\
0 / 15\end{array}$ & GA & 6.0 & 18( & 3878 & $648(652)$ & $1489(20$ & $6832(6276)$ & $\infty 5$ & $0<15$ \\
\hline PSO & 1741( & 3259 & $\infty$ & $\alpha$ & 0 & $\alpha$ & $\infty$ & $0 / 15$ & PSO & & & & & & & & \\
\hline $\mathrm{ABC}$ & 2.1e4(2e4) & $\infty$ & $\infty$ & $\infty$ & $\infty$ & $\infty$ & $\infty 5 e$ & $0 / 15$ & $\mathrm{ABC}$ & $5.1(5)$ & $7.6(9)$ & $35(65)$ & $237(149)$ & $374(434)$ & $3312(3293)$ & 6897(7959) & $0 / 15$ \\
\hline$\Delta f_{\text {opt }}$ & 1e1 & $1 \mathrm{e} 0$ & $1 e-1$ & $1 e-2$ & $10-3$ & $1 e-5$ & & \#succ & $\Delta f_{\text {opt }}$ & $1 \mathrm{e} 1$ & $1 \mathrm{e} 0$ & $1 \mathrm{e}-1$ & $1 \mathrm{e}-2$ & $1 \mathrm{e}-3$ & $1 e-5$ & $1 e-7$ & \#succ \\
\hline f11 & & & & & & & & $15 / 15$ & f23 & 3.0 & 518 & 14249 & 27890 & 31654 & 33030 & 34256 & $15 / 15$ \\
\hline SSEABC & $8.5(0.8)^{\star}$ & $7.2(2)^{\star 4}$ & $2.1(0.2)^{\star 4}$ & $\mathbf{1 . 8}(0.1) \star 4$ & $\mathbf{1 . 6 ( 0 . 1 ) \star 4}$ & $1.4(0.1) \star 4$ & $1.3(0.1)^{\star 4}$ & & SSEABC & C $2.6(4)$ & $10(8$ & $6.1(7)^{\star}$ & $25(32)^{\star}$ & $22(25)^{\star}$ & $21(34)^{\star}$ & $21(17)^{\star}$ & $1 / 15$ \\
\hline $\mathrm{GA}$ & $338(56$ & $7124(4$ & $9351(8$ & $\infty$ & $\infty$ & (0.1) & & $\mid \begin{array}{l}15 / 15 \\
0 / 15\end{array}$ & GA & & & & $\infty$ & $\infty$ & $\infty$ & $\infty 5 e 5$ & 15 \\
\hline PSO & $91(57)$ & $235(125)$ & $123(68)$ & $140(63)$ & $164(64)$ & $243(46)$ & $391(26$ & $\begin{array}{l}\text { o/15 } \\
8 / 15\end{array}$ & PSO & $2.2(2)$ & $20(23)$ & $243(224)$ & $\infty$ & $\infty$ & $\infty$ & $\infty 5 e 5$ & $0 / 15$ \\
\hline $\mathrm{ABC}$ & $160(54)$ & $6082(2450)$ & 9334(5734) & $\infty$ & $\infty$ & $\infty$ & $\infty 5 e 5$ & $\mid \begin{array}{l}0 / 13 \\
0 / 15\end{array}$ & $\mathrm{ABC}$ & $2.2(2)$ & $19(27)$ & $\infty$ & $\infty$ & $\infty$ & $\infty$ & $\infty 5 e 5$ & o/15 \\
\hline$\Delta f_{\text {opt }}$ & $1 \mathrm{e} 1$ & $1 \mathrm{e} 0$ & $1 \mathrm{e}-1$ & $1 e-2$ & $1 e-3$ & $1 e-5$ & $1 e-7$ & \#succ & $\Delta f_{\text {opt }}$ & 1e1 & & & & $1 \mathrm{e}$ & $\begin{array}{llll}e 0 & 1 e-1 & 1 e-2 & 1\end{array}$ & $\begin{array}{ll}1 e-3 & 1 e-5 \quad 1 e-7\end{array}$ & \#succ \\
\hline f12 & 108 & 26 & 371 & 413 & 461 & 1303 & 1494 & $15 / 15$ & f24 & & & 1622 & & 2.2 & $2 \mathrm{e} 56.4 \mathrm{e} 69.6 \mathrm{e} 69$ & $9.6 \mathrm{e} 61.3 \mathrm{e} 7 \quad 1.3 \mathrm{e}$ & $3 / 15$ \\
\hline SSEABC & $7.2(0.5)^{\star 3}$ & 7.4(1 & $11(6)^{\star 3}$ & $13(6)^{\star 3}$ & $17(20)^{\star 3}$ & $8.3(8)^{\star 4}$ & & $12 / 15$ & $\begin{array}{l}\text { SSE } \\
\text { GA }\end{array}$ & $\begin{array}{c}C \\
\text { C.8.8(2) }\end{array}$ & & & & & $\infty \quad \infty$ & $\infty \quad \infty \quad \infty 5 e 4$ & $40 / 15$ \\
\hline GA & $933(1262$ & $2447(2403)$ & $1.9 \mathrm{e} 4(1 \mathrm{e} 4)$ & $\propto$ & $\infty$ & $\infty$ & & & $\begin{array}{l}\text { GA } \\
\text { PSO }\end{array}$ & & & & & $\infty_{\infty}^{\infty} 1-1$ & $\begin{array}{lll}0 & \infty & \infty \\
0 & \infty & \infty\end{array}$ & 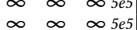 & 5 \\
\hline PSO & $748(1162)$ & $3747(8396)$ & $5406(9771)$ & $7886(1 \mathrm{e} 4)$ & $1.5 \mathrm{e} 4(2 \mathrm{e} 4)$ & 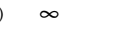 & $\infty 5 e 5$ & $0 / 15$ & $\mathrm{ABC}$ & $13(20)$ & & & & & $0 \quad \infty \quad \infty$ & 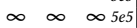 & $500 / 15$ \\
\hline
\end{tabular}

Table 2: Average runtime (aRT in number of function evaluations) divided by the respective best aRT measured during BBOB2009 in dimension 5 . The aRT and in braces, as dispersion measure, the half difference between 10 and $90 \%$-tile of bootstrapped run lengths appear for each algorithm and target, the corresponding reference aRT in the first row. The different target $\Delta f$ values are shown in the top row. \#succ is the number of trials that reached the (final) target $f_{\text {opt }}+10^{-8}$. The median number of conducted function evaluations is additionally given in italics, if the target in the last column was never reached. Entries, succeeded by a star, are statistically significantly better (according to the rank-sum test) when compared to all other algorithms of the table, with $p=0.05$ or $p=10^{-k}$ when the number $k$ following the star is larger than 1 , with Bonferroni correction of 110. A $\downarrow$ indicates the same tested against the best algorithm from BBOB 2009. Best results are printed in bold. 


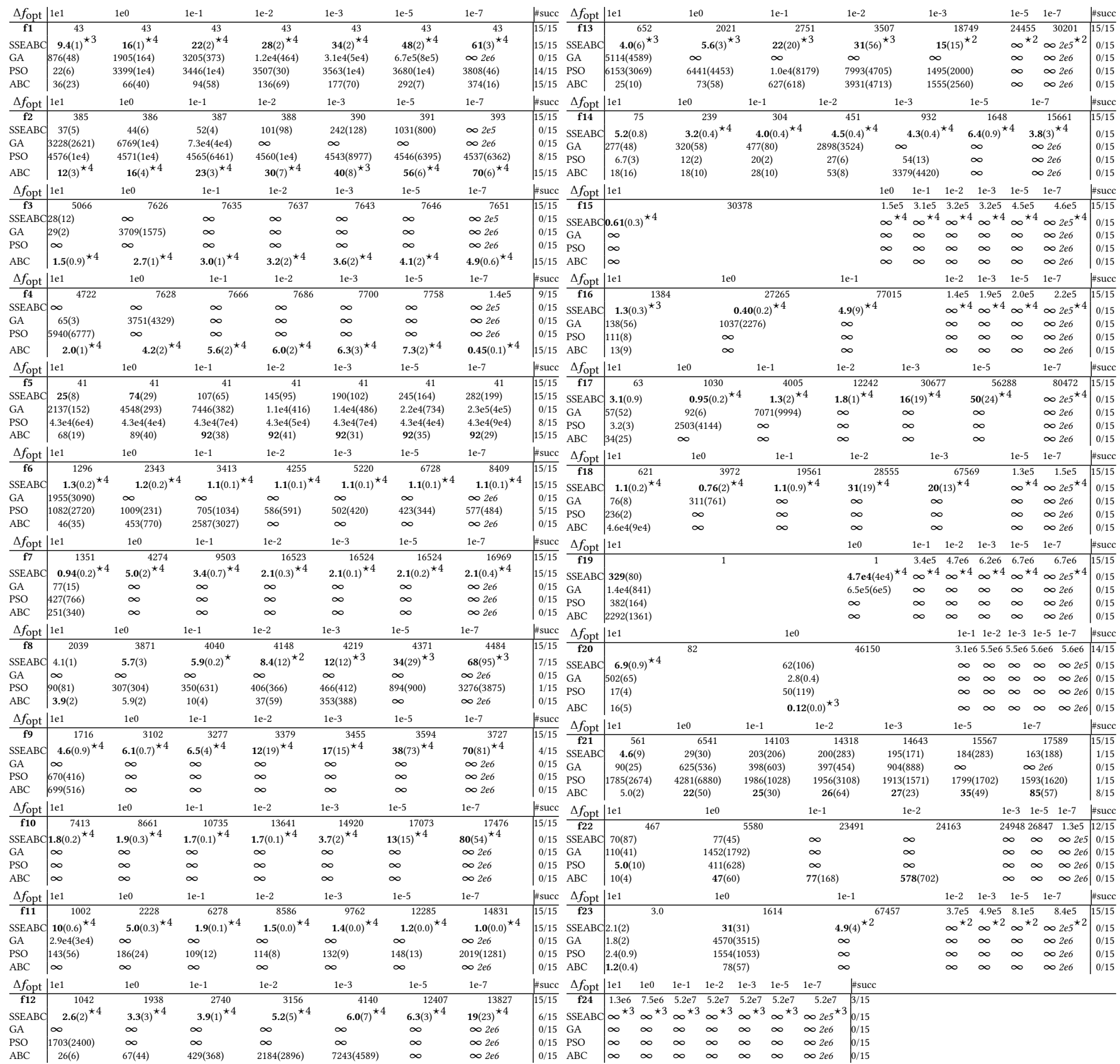

Table 3: Average runtime (aRT in number of function evaluations) divided by the respective best aRT measured during BBOB2009 in dimension 20 . The aRT and in braces, as dispersion measure, the half difference between 10 and $90 \%$-tile of bootstrapped run lengths appear for each algorithm and target, the corresponding reference aRT in the first row. The different target $\Delta f$ values are shown in the top row. \#succ is the number of trials that reached the (final) target $f_{\text {opt }}+10^{-8}$. The median number of conducted function evaluations is additionally given in italics, if the target in the last column was never reached. Entries, succeeded by a star, are statistically significantly better (according to the rank-sum test) when compared to all other algorithms of the table, with $p=0.05$ or $p=10^{-k}$ when the number $k$ following the star is larger than 1 , with Bonferroni correction of 110. A $\downarrow$ indicates the same tested against the best algorithm from BBOB 2009. Best results are printed in bold. 